\title{
LA CARTOGRAFÍA EN SALUD PÚBLICA: VIEJOS PROBLEMAS Y NUEVAS OPORTUNIDADES
}

\section{Luisa Iñiguez ${ }^{1}$ \\ Christovam Barcellos ${ }^{2}$}

La división convencional de la geografía de la salud en geografía de problemas o daños a la salud, también conocida como Nosogeografía (Darchenkova, 1988), y geografía de la atención médica o de los cuidados a la salud, puede extenderse a la bifurcación de la cartografía de la salud en dos áreas de su desarrollo. Así, por lo general los mapas identifican distribuciones espaciales de problemas de salud y sus determinantes, o sustentan la distribución de los servicios de atención según necesidades en salud; la articulación de estas dos direcciones es aún muy escasa.

Un nuevo escenario de la cartografía en salud, se ha creado por el notable desarrollo de las técnicas geoprocesamiento y del análisis espacial en salud, y los avances en el reconocimiento de la utilidad de los mapas en la gestión del sector salud. La cartografía incrementa así sus potencialidades para "conocer e interpretar y para actuar".

Ello es constatado tanto en publicaciones, como en su empleo en direcciones municipales o unidades de servicios, en la organización de la vigilancia, en programas de control de problemas de salud, y en la distribución o redistribución de servicios o equipamientos especializados. Muchos gobiernos locales invierten en la creación de bancos de datos de utilización común entre sectores de salud y otros para la gestión municipal integrada. Algunos países disponen datos de salud en forma de mapas web para visualización de condiciones de salud y la vigilancia de enfermedades. La disponibilidad de datos, junto a la creciente facilidad de empleo de programas de SIG, ha creado en las últimas décadas condiciones para el análisis de datos espaciales, que antes eran limitadas a los ambientes académicos.

Todo este proceso reciente de apropiación de técnicas de cartografía y popularización de concepciones geográficas de los problemas de salud no superan las dificultades metodológicas

\footnotetext{
${ }^{1}$ Facultad Latinoamericana de Ciencias Sociales. Grupo de Estudios de Salud y Bienestar Humano. Universidad de La Habana. e-mail: luisa@flacso.uh.cu

${ }^{2}$ Laboratório de Informação em Saúde, Fundação Oswaldo Cruz e-mail: xris@fiocruz.br
} 
involucradas en la concepción y confección de mapas de la salud, o para la salud. Al contrario, frente a la profusión de técnicas y de datos, queda cada día más compleja la tarea de representar los problemas de salud en mapas. El presente artículo pretende exponer algunas reflexiones acerca de la evolución reciente de la cartografía en el campo la salud y de la salud pública, colocando el foco en las soluciones propuestas a retos antiguos, y a otros nuevos surgidos dentro y fuera de la geografía de la salud.

\section{La cartografía en salud. Las áreas y los puntos}

Hoy día asistimos a una transformación de las prácticas de Salud Pública con el uso de sistemas de información - que registran datos vitales, enfermedades y consumos de servicio de salud - y la incorporación de técnicas de georreferenciación a la rutina de la vigilancia, apoyada a su vez en el desarrollo de bases cartográficas digitales en el sector salud y en los múltiples sectores que integran la Salud Pública. Es cada vez más fácil y posible crear mapas de eventos de salud para identificar riesgos, o mapas de distribución de servicios de atención, con vistas a subsidiar estrategias para el control de daños a la salud, o la reorganización servicios de atención.

La tecnología disponible permite colocar juntos en un mapa objetos dispares y procedentes de diversas fuentes, tales como postes de iluminación, parques públicos, superficies acuosas, manzanas de la trama urbana, viviendas pobres, hospitales, viales, defunciones, focos de mosquitos, casos de enfermedades transmisibles, centros de atención a la salud, o áreas tributarias a los centros de salud. La geografía frente a esta nube de puntos y una malla de polígonos, es capaz de darle un sentido a los inmensos volúmenes de información, con recursos científicos y tecnológicos, que interese al campo de la salud y de la Salud Pública (Barcellos, 2007).

Este reto es teórico-conceptual, porque obliga a pensar las posibles relaciones entre objetos, y a la vez es técnico-metodológico, porque exige el uso de herramientas que auxilien la búsqueda de patrones de distribución espacial en medio de una aparente profusión de puntos o de concentración de áreas con similares valores. 
Dos caminos son posibles y recomendables: Entender el lugar y sus recortes para explicar la distribución espacial de eventos, o buscar patrones espaciales de eventos, para comprender los determinantes socio-ambientales de su distribución en un área dada.

\section{De puntos a áreas. Los agregados predeterminados.}

La forma más usual de evaluar eventos o riesgos en salud ha sido agregar en unidades administrativas predeterminadas, datos producidos por el sector y datos demográficos, que posibilitan el cálculo de indicadores epidemiológicos. De forma general estos datos son siempre puntuales, se registran al interior de cada unidad espacial discreta y se asignan convencionalmente a ellas. Así, los mapas más frecuentes, están referidos a áreas (polígonos) intencionalmente seleccionados, que constituyen territorios político administrativos sub nacionales o de la administración del sector salud, tales como provincias (estados), municipios, áreas con población que tributa a un centro de salud, a consultorios del médico de la familia, o a áreas de atendidas por los equipos de Programa de salud de la Familia, entre otras.

Ambos datos agregados, "de eventos y de población" colectados al interior de cada área, permiten el cálculo de tasas generales o específicas, brutas o estandarizadas, razones, porcentajes, probabilidades, u otras, usualmente redactados como mapas coropléticos. Si bien esta rutina continúa como vía para facilitar la comparación entre las unidades territoriales, y conocer la distribución de eventos de salud, ambos agregados de información portan varios inconvenientes.

Entre ellos cabe destacar la heterogeneidad ambiental y social interna, las variaciones en el área y la cantidad de población de las unidades espaciales y la propia distribución de la población al interior del área. Dos hipótesis convencionales sustentan la elaboración de mapas coropléticos: las áreas delimitadas son internamente homogéneas, uniformes y el fenómeno representado existe en todos los puntos de esas áreas; el evento a representar tiene una intensidad media en todos los puntos del área (SORRE, 1978 ${ }^{3}$ ).

Las limitaciones que imponen estas hipótesis son evidentes. Tanto en los recortes político administrativos, como en otros recortes del sector salud, la heterogeneidad interna se vuelve

${ }^{3}$ Citado por Curto de Casas 2003. Mapas en Geografía Médica. 
decisiva en el conocimiento solo aproximado de la distribución del evento dado, lo cual puede provocar que las decisiones de actuación sean erróneas. Una de las respuestas a estas limitaciones es el uso de cartodiagramas o la representación por el proceso de anamorfosis.

Otra limitación es la no consideración de interacciones entre estas unidades espaciales y la inestabilidad de los indicadores para áreas pequeñas (King, 1979), que en realidad se refiere a poblaciones pequeñas, con independencia de la superficie de estas. La adopción de uno u otro recorte espacial (cuencas, municipalidades, ecosistemas, y otros.) representan una aproximación a la totalidad geográfica, y esta selección puede influenciar los resultados visuales y estadísticos obtenidos. Algunas técnicas de análisis se han desarrollado con el objetivo de considerar las relaciones de proximidad o vecindad entre unidades espaciales, entre ellas la aproximación bayesiana para estimación de valores de tasas, que reduce la variabilidad local y rescata la estructura espacial de dependencia entre valores próximos (Auchincloss et al., 2013). Al conocimiento impreciso de la distribución, y la interpretación que de estos mapas puede hacerse, se integra su principal restricción que deviene de las posibilidades de actuación a partir de ellos, al sugerir que la intensidad del evento representado se distribuye de forma homogénea dentro de cada área, lo cual tiene implicaciones más serias en el caso de la distribución de servicios o recursos humanos por habitantes, o en la propia densidad bruta de población, que desconoce las áreas inhabitadas.

Con el doble propósito de encontrar unidades espaciales más homogéneas, y suplir la falta de información de contextos de vida en las estadísticas sanitarias, comenzó a ser cada vez más frecuente el uso de unidades censales (sector, distrito u otras). Como ha sido mostrado (Barcellos, 2003), también existe heterogeneidad al interior de estas pequeñas unidades, aunque es indudable que las distribuciones se precisan. Por otra parte, la temporalidad de los datos censales, producidos como norma cada 10 años, se vuelven obsoletos en poco tiempo, en especial en zonas de elevado dinamismo económico o social y en consecuencia poblacional.

Otro de los retos mantenidos es la no adecuación de los sectores censales, a otros recortes del sector salud. En estudios acerca de mapas participativos como alternativa cartográfica para la estrategia de salud de la familia (ESF), fue probado que el diseño de áreas sobre mapas de sectores censales, puede auxiliar el proceso de organización del trabajo de los equipos del ESF. No obstante, se señala como limitante, la escasa coincidencia cartográfica entre los límites de 
los sectores censales y las áreas delimitadas por la ESF, aunque en unidades de mayor jerarquía, tienden por lo general a no ultrapasar los límites de barrios o distritos. (Goldstein et al, 2013) Para responder al "problema de las unidades de área modificables" (Openshaw, 1984) creado por la imposición de unidades espaciales artificiales que intentan delimitar fenómenos geográficos continuos, se incorpora también como estrategia alternativa la creación de grandes "áreas homogéneas". Las técnicas de estadística multivariada pueden ser útiles para agrupar pequeñas áreas a partir del análisis de un conjunto de variables socio-ambientales (Buzai, 2003).

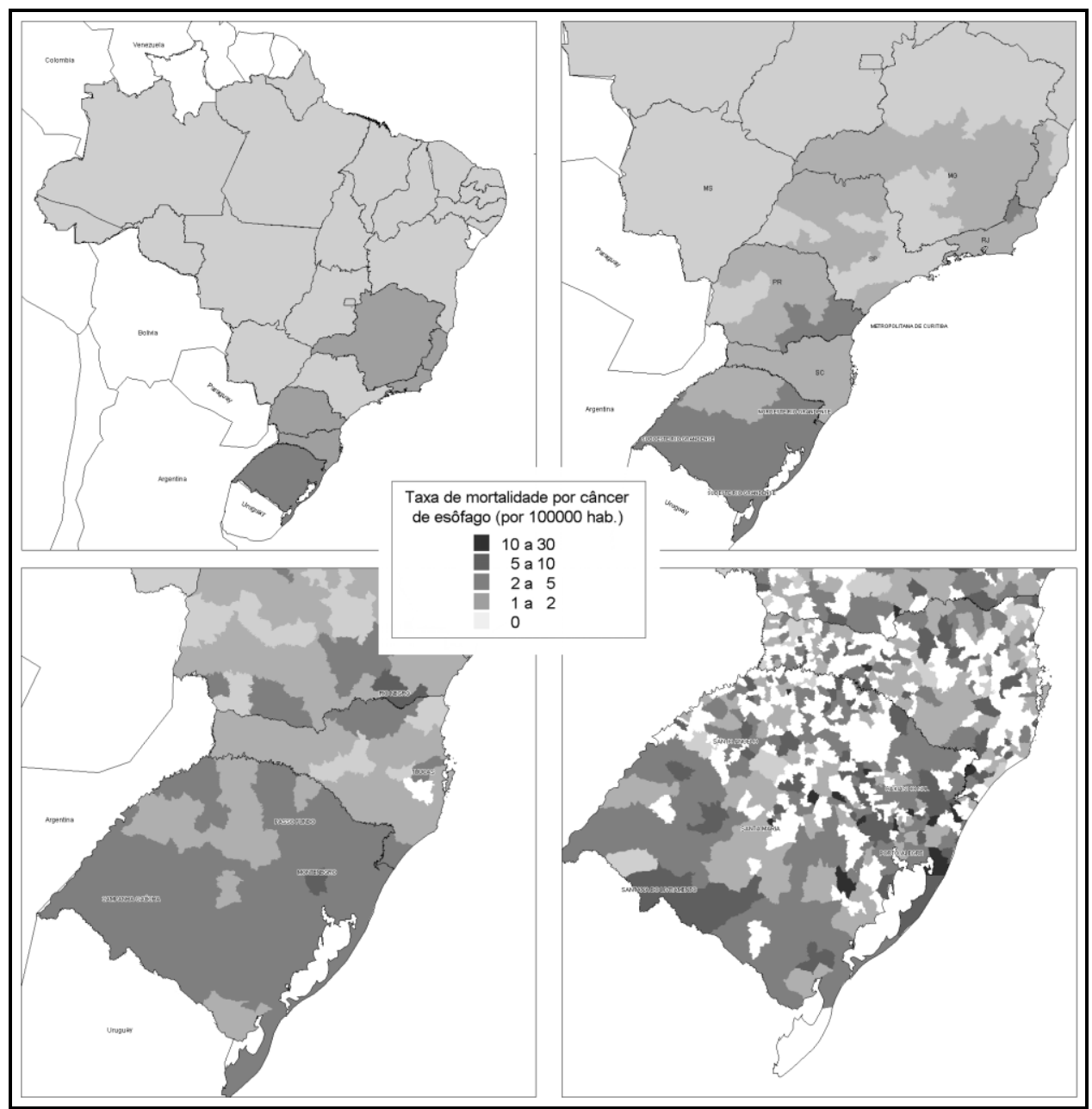

Figura 1: Efecto de la agregación de datos en unidades espaciales. El caso de la mortalidad por cáncer de esófago en Brasil (Barcellos, 2003).

De los puntos a las áreas. Los agregados inducidos 
Una de las formas tradicionales de elaborar mapas de interés para la Salud Pública es la representación de eventos en forma de puntos, los cuales en el momento de construcción de la base de datos geográficos y durante el procedimiento de análisis, minimiza problemas asociados a la adopción de áreas administrativas artificiales (Dunn et al. 2001). Esta estrategia fue utilizada por John Snow en el famoso y pionero mapa del brote de cólera en Londres en 1854. En este caso, no había a priori una zona (polígono) sospechoso de riesgo, sino un conjunto de puntos, cuyo patrón podría mostrar una concentración de riegos.

El caso más sencillo de construcción de tasas para zonas no administrativas es la creación de áreas de influencia (buffer) alrededor de una fuente sospechosa de exposición. También en este caso se pueden calcular tasas de incidencia dentro y fuera del área de influencia y la significación estadística de la diferencia entre tasas. Si estas áreas de influencia se forman a partir de círculos concéntricos, se puede evaluar si los riesgos decaen con el aumento de la distancia a la fuente sospechosa (Michelozzi et al., 2002).

La localización de eventos de salud es interés especial para la vigilancia, tales como los accidentes de tránsito, casos de sarampión o internaciones; si estos están concentrados en el espacio es posible que exista una determinación socio-espacial del evento. Encontrar los lugares de mayor incidencia constituye un indicio para identificar factores ambientales, sociales o vinculados a la propia asistencia a la salud.

La primera pregunta formulada frente a una nube de puntos es ¿existe un patrón espacial de distribución? O traduciéndola como prueba estadística, ¿el patrón de distribución de puntos es diferente del aleatorio y del regular? El ambiente SIG permite con facilidad calcular matrices de distancia entre puntos y a partir de esta producir estadísticas.

Estos métodos, basados en la medición de distancia entre puntos, indican áreas donde se concentran casos, pero por su naturaleza, ellos tienden a una distribución espacial semejante a la propia distribución de población. Todos los eventos de salud - el nacimiento, la infección, la enfermedad, la internación, la muerte - se manifiestan en las personas. Y estas personas no están distribuidas aleatoriamente en el espacio, sino siguen tendencias históricas y socioeconómicas.

Por ello, al trabajar con registros de problemas de salud para evaluar riesgos se debe estimar la probabilidad de ocurrencia del evento, ponderada por la distribución de población, o sea la 
densidad demográfica. La pregunta en este caso se convierte en: ¿La distribución espacial de puntos es diferente de la distribución de la población? Las preguntas formuladas son reflejos de las hipótesis de investigación para las cuales han sido desarrolladas distintas técnicas estadísticas (Waller, 2000).

Pero no siempre la fuente de exposición tiene la forma de punto. En gran número de situaciones, la fuente suele ser una línea (una carretera que emite gases dañosos, por ejemplo) o un conjunto de manchas (focos de mosquito), en el caso de fuentes múltiples. Las técnicas de interpolación permiten construir zonas a partir de datos ambientales puntuales (como por ejemplo la contaminación del aire) para por encima de estas zonas calcular tasas de incidencia y la razón entre tasas.

La identificación del patrón de distribución de casos puede ser un indicio para identificar fuentes de exposición desconocidas, ya que casos próximos suelen compartir condiciones ambientales semejantes. La matriz de distancias entre puntos puede ser usada para la construcción de jerarquías de conglomerados, asumiendo la distancia entre puntos vecinos como medida de disimilitud. Son parámetros importantes para este cálculo la distancia umbral, que limita la probabilidad de seleccionarse un par de puntos por casualidad y el número mínimo de puntos necesarios para constituirse un conglomerado. Esta rutina de cálculos permite el diseño de elipses que encierran conglomerados de casos (Levine, 2002).

Otro interesante método de análisis es derivado de las técnicas de suavización y interpolación de Kernel o de kriging para suavización de puntos, generando una superficie de densidad para la identificación visual de "áreas calientes" (Gatrell et al. 1996). La aplicación de la estimativa Kernel en Salud Pública está basada en el cálculo de densidad de casos (número de eventos por área), que produce una superficie de probabilidades de ocurrencia donde áreas con casos más cercanos presentan mayor densidad (Santos et al. 2001). La técnica permite identificar áreas la concentración de accidentes de tránsito, o de focos de vectores, para establecer medidas de control y para la estimativa de tasas de incidencia. En este caso, la superficie de densidad de casos es dividida por la densidad de población (habitantes por área) también como superficie continua, produciendo una tercera superficie que representa la probabilidad de encontrar casos en una nube de habitantes, esto es la tasa suavizada de incidencia. 
En situaciones donde no se dispone de datos de población se puede probar la aleatoriedad de "casos" con relación a un conjunto de "controles", obtenidos por encuesta o una muestra de una población de perfil semejante.

Una técnica, llamada barredura o scan, permite que se evalúe la incidencia de casos dentro de un círculo que camina sobre el espacio estudiado. El círculo cambia su radio y posición con el objetivo de buscar el mejor circulo que presente diferencias significativas entre tasas de incidencia dentro y fuera de ese círculo (Kulldorff y Nagarwalla, 1995). Comparada con otros métodos estadísticos espaciales, la estadística scan presenta ventajas para la identificación de conglomerados: se puede ajustar el efecto de diferentes densidades de población, puede incorporar otras variables que caracterizan el perfil socio-económico de las poblaciones, y puede identificar conglomerados, sin una localización presumida a priori.

Es importante subrayar que el propio proceso de análisis produce nuevas capas, o objetos geográficos, que son derivados de observaciones individuales, pero constituyen polígonos, o zonas, con población, características geofísicas, ubicación y una representación simbólica de riesgos.

Una vez identificadas las zonas de más alta incidencia, cualquiera sea el método utilizado, el paso siguiente es calcular indicadores socio-ambientales que expresen los determinantes de salud en el espacio geográfico. La pregunta se convierte en: ¿Hay diferencias sociales y ambientales entre áreas de alta y baja incidencia?

La Geografía tiene como contribución en este debate, buscar evidencias de asociación entre la concentración de eventos y sus determinantes socio-ambientales en el espacio, que no es una superficie lisa, sino una trama de complejas relaciones entre objetos y acciones (Santos, 1997). Cabe en este caso, identificar y localizar estos determinantes para estudiar como ellos condicionan la distribución de casos (Rushton, 2003).

Las posibles localizaciones de los puntos según los sistemas de información disponibles, dependen del objetivo propuesto. Así son redactados mapas según lugar de tratamiento, lugar de residencia, lugar donde ocurrieron las muertes y lugares probables de infección. En algunas entidades nosológicas que por lo general tienen prolongada evolución sub clínica, la posibilidad de identificar riesgos, se reduce y la dirección de residencia, puede no informar sobre el lugar 
probable de infección, al contrario sucede con las entidades de súbita presentación de síntomas, al contacto con el agente infeccioso.

Otra posibilidad de la localización de puntos es la construcción a partir de ellos de mapas de flujos, que por ejemplo unen el lugar de la institución donde se recibe el tratamiento con el lugar de residencia de los beneficiarios, donde el grosor muestra la intensidad del flujo de pacientes. Las técnicas de análisis espacial permiten diseñar superficies con similares condiciones de accesibilidad a los servicios de salud, a partir de puntos que representan todos los lugares poblados, y consideran el relieve, los tipos de viales y las superficies acuosas, para aproximarse lo más posible a las barreras en la movilidad de los individuos. En la Fig. 2 a se muestran los casos de VIH/SIDA por símbolos proporcionales, y los flujos de los pacientes a las instituciones de salud donde son atendidos por líneas. En la Fig.2 b se muestran las dificultades de acceso, de la población de todos los asentamientos de un municipio al policlínico donde deben recibir atención primaria, dividido en cinco rangos de menor a mayor dificultad. Ambos mapas aportan resultados de interés para la organización y las estrategias de atención del sector salud.
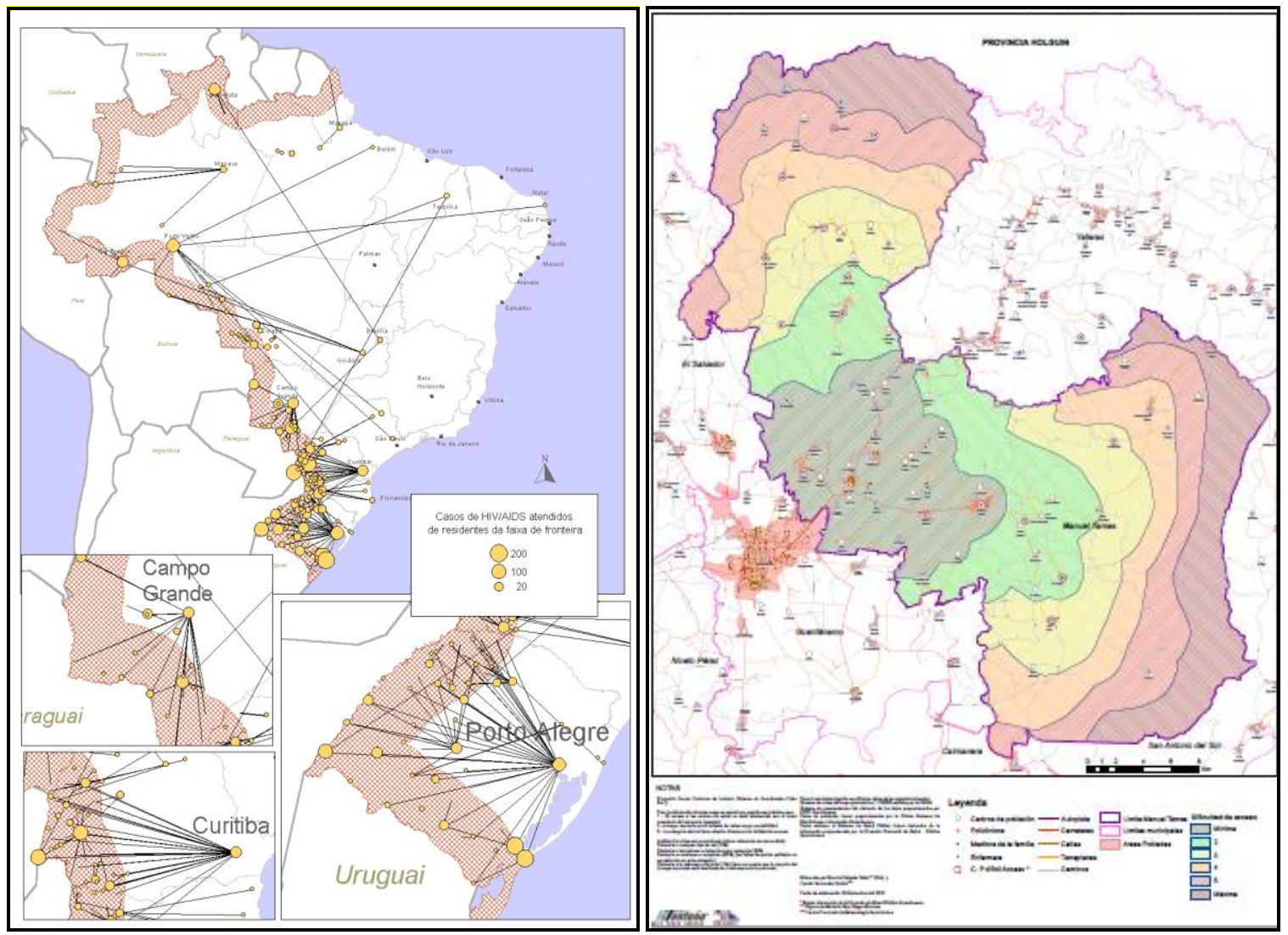
Figura 2 (a) Flujo de atenciones de residentes en las áreas de frontera de Brasil. Fuente: CN DST/Aids, (Barcellos, 2000 org.); (b) Accesibilidad al policlínico en el municipio de Manuel Tames, Provincia de Guantánamo. 2013 Cuba (Hernández, C org.).

A pesar de su potencial, para la localización o re localización de unidades de atención, equipamientos, o recursos humanos, o para medir el nivel de utilización de los servicios, o la difusión de un evento, en nuestro contexto regional, estos mapas son muy escasos.

Los geógrafos interesados en la cartografía en salud, deben unir las dos tendencias de los análisis espaciales, la exploración de patrones ("pattern spotters and testers") y la búsqueda de relaciones ("relationship seekers and provers"), como bien las nombró Openshaw (1994).

\section{De los mapas tradicionales a los mapas actuales en salud.}

Al ¿dónde?, pregunta inicial, y muchas veces casi única, en los mapas en el campo de la salud, lo acompañan en el contexto del desarrollo científico y tecnológico otras interrogantes. Los mapas que describen o constatan distribuciones son aún importantes, aún mantienen su vigencia, y a pesar de sus inexactitudes, hasta el sencillo mapa de distribución de puntos aleatorios dentro de unidades político administrativas, sugiere de manera inmediata anomalías a quien conoce la distribución de la población en un territorio dado.

El ¿cuándo? de larga tradición en la cartografía de enfermedades, representado por lo general por un conjunto de mapas de casos y tasas de "ciclo epidémicos", o de difusión de entidades infecciosas, usualmente referidas a semanas epidemiológicas, cuenta en la actualidad con recursos que incluso permiten la localización "en tiempo real" de registros de entidades de notificación compulsoria o emergente vigiladas.

En tanto las posibilidades ampliadas de responder al ¿por qué? de las distribuciones halladas, ha posibilitado avanzar de las descripciones a los procesos de producción del evento, en especial en el caso de las enfermedades infecciosas y parasitarias a expensas del desarrollo de otras ciencias como la biología molecular; los registros de casos son más precisos y confiables dada la organización de diferentes sistemas de información y el aumento de la cobertura de instituciones de atención a la salud; crece la disponibilidad de diferentes bases de datos georreferenciadas y los recursos para georreferenciar, así como se evidencia un notable 
aumento de personas preparadas e interesadas en el tema. En ese escenario el geoprocesamiento se posiciona como herramienta esencial para la organización y análisis de los datos, en especial a partir de los Sistemas de Información Geográfica (SIG), una de sus vertientes (Croner et al., 1996).

Por ejemplo, es posible superponer mapas de localización equipamientos urbanos sobre áreas urbanas degradadas, e identificar grupos socio -espaciales excluidos, caracterizados por sus condiciones socio económicas y por la dificultad de acceso a los servicios de salud. Esa operación entre capas crea una nueva información que sería de difícil obtención mediante tablas, o de forma manual como por tradición era efectuado.

Las técnicas de análisis espaciales en Salud Pública han reforzado la atención a la identificación de riesgos. Así como existen varias maneras de conceptuar y cuantificar riesgos, son múltiples los usos para su determinación. Un mapa de riesgo se puede construir, por ejemplo, apuntando la presencia de factores socio-ambientales de riesgo, como áreas de inundación, de pobreza extrema o de contaminación industrial. También se concibe como un mapa de riesgo, la medición de frecuencia de eventos adversos para la salud.

Por ejemplo, un mapa de tasas de mortalidad infantil del pasado se aproxima de la distribución de los riesgos de morir en la niñez en el futuro. Estas diferentes definiciones de riesgo, una de las Ingenierías, otra de la Estadística, se complementan con la concepción epidemiológica según la cual el riesgo es la medida de tendencia a formar conglomerados de casos entre poblaciones expuestas, comparadas con poblaciones no expuestas.

El creciente uso del geoprocesamiento en el área de la salud ha aumentado la capacidad de formular y evaluar hipótesis sobre la distribución espacial de problemas de salud, principalmente mediante la confección relativamente rápida de mapas temáticos. Pero, estos procedimientos no siempre son suficientes por tratarse de datos espacial de elevada complejidad. En esos casos es necesario una etapa de modelación y análisis espacial de datos que están asociados a diferentes capas y temas (Barcellos, 2007). Se reconoce que los SIG tienen un rol central en el modelaje de datos espaciales, a través de operaciones de superposición de capas sobre la incidencia de eventos de salud a otras capas de interés (Briggs y Elliott, 1995). 
En la actualidad mediante modelos de análisis espacial en ambiente de SIG, es posible establecer y analizar escenarios para la ocupación de un territorio, con la simulación de diversas condiciones de urbanización y ambientales.

\section{La leptospirosis urbana como caso.}

Se estudió la distribución espacial de los casos de leptospirosis ocurridos en la década de 1990 no municipio de Porto Alegre (Brasil), combinando factores ambientales e individuales. La primera etapa esencial para la construcción del mapa fue la georreferenciación de los 172 casos notificados en el periodo según el lugar presunto de infección. La segunda etapa es la reunión de otros datos espaciales (capas) que permitan recuperar el contexto en el cual se produjeron los casos. Datos socio-demográficos fueron obtenidos según los sectores censales, lo que permitió el mapeo de condiciones de ingreso, habitación, saneamiento y población. Otras informaciones como relieve y vegetación fueran obtenidas por medio del "Atlas Ambiental de Porto Alegre" (Menegat, 1999).

La tercera etapa, no menos critica, se construye una basis común dentro de la cual se van añadir todos los datos anteriores. En este caso, se optó por un grid de 250 metros de largo que cubre todo el territorio del municipio. La variable respuesta es la presencia de casos en cada una de las 7600 celdas, que se busca explicar por medio de variables auxiliares (ambientales y sociales) también referentes a las celdas. Un modelo de regresión logística fue utilizado para medir la asociación entre estos grupos de variables. El esquema de análisis es resumido en la figura 3. 


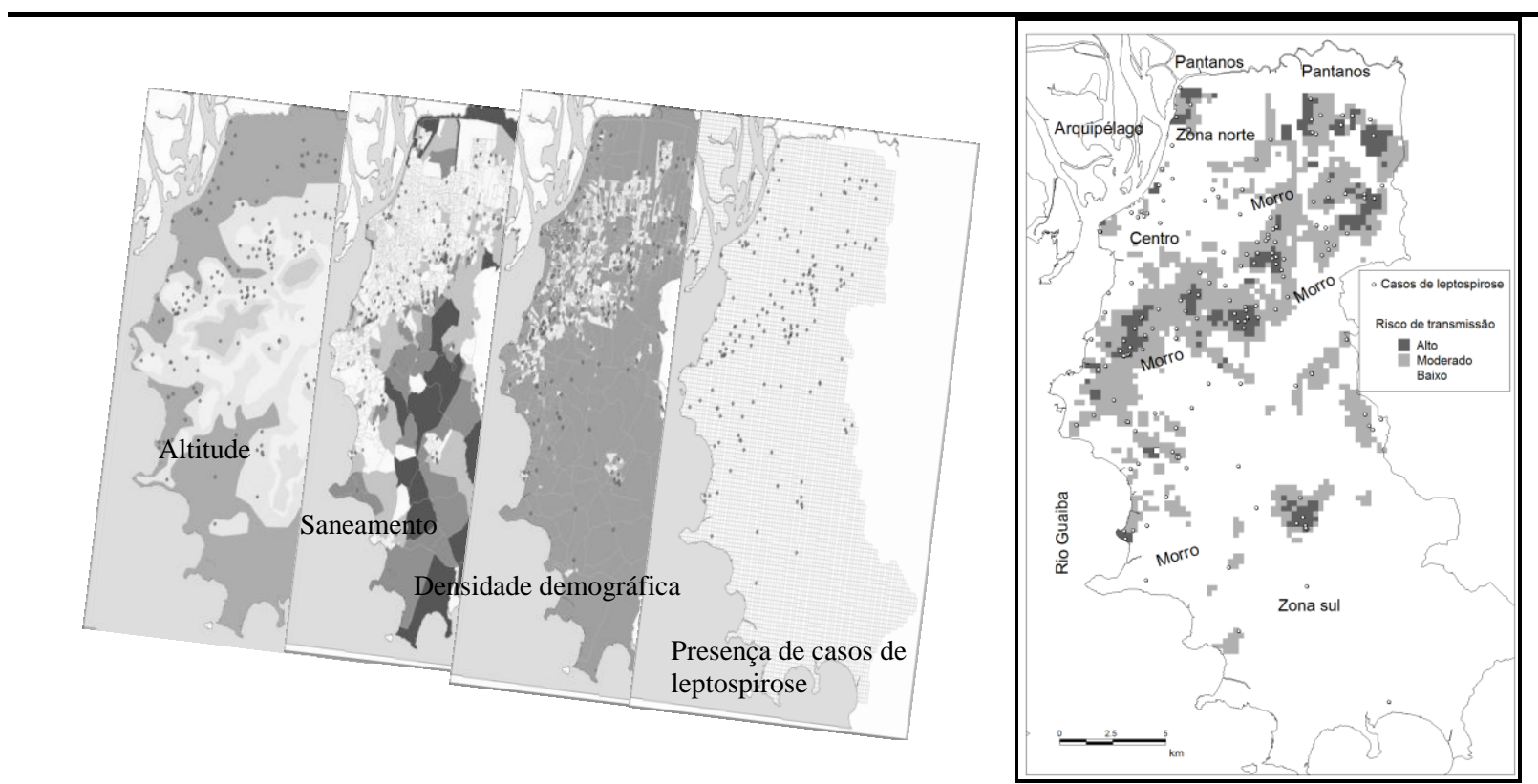

Figura 3 (a): Capas de datos usados para el análisis de la distribución de casos de leptospirosis. (b): Ubicación de casos de leptospirosis según el local presunto de infecçion y locales de riesgo de transmision de la enfermaedad segun el modelo.

El modelo fue capaz de predecir en torno al $30 \%$ de las celdas observadas como positivas (conteniendo casos), y fue encontrado un ajuste razonable entre valores observados y previstos por el teste de chi-cuadrado. El modelo presento alta sensibilidad, pero baja especificidad. Por ello se propone su refinamiento y la inserción de variables que indiquen la presencia de animales peri domiciliares, y un mayor detalle de los tipos de vegetación en el municipio. Las principales variables explicativas seleccionadas para el modelo son la densidad demográfica, el bajo ingreso, la presencia de casas (y no apartamentos), la baja cobertura de servicios de saneamiento y la proximidad de áreas verdes. La composición entre estas variables representa el contexto en el que se produce la enfermedad.

El riesgo de transmisión, previsto por el modelo de regresión, indicó la ampliación de las áreas de transmisión potencial de leptospirosis en torno a $500 \mathrm{~m}$ de radio alrededor de los casos individuales. Este resultado se permite sugerir que los radios de actuación de los programas de control deben ser extendidos hacia los entornos más amplios que el peridomicilio de los casos. Así, los lugares de concentración de factores de riego pueden no coincidir en el espacio y en el tiempo con los locales de mayor incidencia de casos. Este defasaje sugiere la revisión de modelos de investigación y control de enfermedades a partir de la teoría de la nidalidad y una adecuación de las metodologías estadísticas de tratamientos de datos espaciales, basados en la 
búsqueda de conglomerados de casos o eventos de salud, mediante las técnicas de estadística espacial.

\section{Los mapas en salud. ¿Medios o fines?}

La más crucial de las preguntas en la cartografía en salud es hoy ¿para quién?, los fines y los propósitos de los mapas. Los mapas como ilustración de la ubicación del área de estudio, o los que muestran regularidades de la ocurrencia de eventos, más frecuentes, ceden lugar a los mapas analíticos que producen y testan hipótesis, a los propositivos que argumentan la necesidad de cambios en la distribución de servicios según accesibilidad, y a los predictivos que resultan de los avances en las rutinas de la vigilancia en salud.

La antigua máxima de Sáenz de la Calzada (1956) "La Geografía Médica comienza cuando el mapa acaba", deja el espacio a los mapas que describen, analizan, vigilan y sustentan respuestas del sector salud. Por otra parte, el mapa termina, y pocos minutos después la información representada fue variada. El reiterado reto que impone el desfasaje entre la información que muestra y la respuestas del sector salud o de otros sectores, en especial ante expresiones epidémicas, está potencialmente superado por innovaciones tecnológicas que permiten la redacción de mapas dinámicos, interactivos, y hasta actualizados en tiempo real. Resta ahora que esta nueva cartografía pueda apoyar que las acciones se aproximen lo más posible al tiempo real.

No existen dudas de que el más célebre y admirado mapa en salud es el producido por John Show en su investigación del brote de cólera en Londres 1854, que continua siendo un perfecto ejemplo del uso de mapas para evaluar riesgos de manera integrada.

El trabajo de John Snow, hace poco más de 160 años, permite examinar la articulación del ¿Dónde, con los cuándos, los porqué y para quiénes?. En el Mapa 1 de la segunda edición de su trabajo "Sobre la transmisión del cólera" ${ }^{4}$ publicado en diciembre de 1854, representó con trazos la localización de las defunciones por cólera y localizó las bombas de agua en Londres mediante símbolos. El Dr. Snow había formulado la hipótesis de que el agua podría transmitir el

\footnotetext{
${ }^{4}$ Snow, J. (1990). Sobre a maneira de tramissão do cólera. Abrasco, São Paulo,Rio de Janeiro. 1990
} 
cólera, a partir de sus intensos estudios y trabajos de campo, así como del conocimiento de otras investigaciones sobre el cólera.

Como herramienta de análisis espacial, el mapa buscaba probar la hipótesis, demostrar una concentración de puntos en un área de la ciudad, esto es, que los puntos poseían un patrón de distribución espacial. Si este fenómeno ocurría, las defunciones tenían causas o fuentes comunes, lo dirían los "pattern spotters and testers". Por otra parte, la presencia de pozos de agua, inducía las posibles fuentes de exposición de la población. Al asociar las dos informaciones (defunciones y bombas de agua) consiguió evidenciar que la hipótesis de transmisión del cólera por vía hídrica estaba correcta, como suelen afirmar los "relationship seekers and provers".

En el Mapa 2 que acompaña su trabajo, menos comentado, se representa con colores las líneas que limitan las áreas de la ciudad abastecidas de agua por las compañías Southwark and Vauxhall, Lambert y el área donde se entremezclan ambas compañías, ${ }^{5}$ y los distritos al sur del río Támesis. No se han encontrado referencias a la superposición de los mapas 1 y 2, no obstante está implícito el interés del Dr. Snow por diferenciar áreas de riesgo según fuentes de abasto, con el objetivo de proponer a las autoridades locales, como lo hizo, intervenciones en la trama causal de la epidemia, con el cierre de la bomba comprometida (Broad Street).

Epígrafes del contenido del documento citado, sugieren un proceso que denominamos en la actualidad superposición de capas: "Prueba de este modo de transmisión en los aglomerados poblacionales más pobres"; "Relación entre la menor o mayor tasas de mortalidad por cólera en Londres y la menor o mayor elevación del nivel del suelo"; "El efecto del abastecimiento de agua en la mortalidad por cólera entre habitantes de asilos y prisiones".

Los mapas de John Snow sobre la ocurrencia del cólera en Londres, lo llevó junto al reverendo Henry Whitehead, a sentar las bases de una nueva ciencia, como un reto a los conocimientos y prácticas sociales tradicionales. Se considera el primer ejemplo del uso de la geografía y de mapas para comprender las causas de la dispersión de una enfermedad. Pero en especial su trabajo generó un desafío político en la implementación de respuestas a problemas de salud y de la Salud Pública en la ciudad, hecho que se repite en la actualidad.

\section{Los retos mantenidos, anulados y los nuevos retos}

\footnotetext{
${ }^{5}$ Según la información aportada por la Oficina de Registros Generales.
} 
La necesidad de que las estadísticas sanitarias, se amplíen con datos sociales de -casos, defunciones, focos y otros-, es un viejo reclamo a los sistemas de información en salud, llevados a la cartografía en salud que por lo general describe la distribución de un hecho, en la población total o de las características individuales de estos por lo general el sexo y un grupo de edad y raras veces, según color de la piel, grupo étnico, u ocupación de los implicados.

En general los sistemas de información sanitaria en base territorial, adolecen aún de datos que faciliten la comprensión de los procesos sociales de producción de un determinado problema de salud, o de salud pública, colectados casi de forma exclusiva por algunos programas de determinadas entidades nosológicas de prioridad internacional o regional, por trabajo de campo, o por Encuestas Nacionales.

No obstante, constituyen sin dudas avances la disponibilidad de bases de datos en la Web de instituciones nacionales que incluso permiten la elaboración de mapas interactivos por los usuarios. A escala internacional, el Observatorio Global de Salud (GHO) de WHO, centra la cartografía de enfermedades tales como influenza, dengue, cólera, y otras, que muestra tanto la distribución actual de estas entidades, como áreas de riesgo perspectivas. Facilita además la elaboración de mapas en áreas geográficas seleccionadas por el usuario, y la localización de informaciones geográficas, instituciones de salud, viales, y otras.

A propósito, mientras en muchos de los países de nuestra región, se han creado estructuras para impulsar la cartografía, los SIG y los análisis espaciales en los Ministerios o Secretarías Nacionales de Salud, y otros, con el apoyo de Organismos Internacionales como la PAHO Y WHO, a escalas sub nacionales, en muchos casos aún no existen recursos técnicos o humanos ni siquiera para sustituir los formatos analógicos por los digitales.

Las oportunidades de interrelacionar datos de diferentes orígenes y formatos en el proceso de redacción de mapas apuntadas por Vine et al. (1997), constituyen una de las ventajas de la cartografía actual aplicada a los servicios de salud y a la Salud Pública. Otro reto que parece reducirse es de la falta de coincidencia espacial o temporal entre datos que deben vincularse, los de salud referidos a la población, y los del ambiente referido a algo externo a esta población y generados por lo general fuera del sector salud, proceso que condiciona la relación entre problemas de salud con sus determinantes. 
Los retos a que se ha enfrentado la cartografía en el sector salud se reducen por el incremento de la capacidad de representación cartográfica de informaciones complejas, de manejar e integrar una sofisticada base de objetos espaciales y de sus atributos, así como por la agudeza analítica que aporta el conjunto de procedimientos de análisis espacial, como propiedades de los SIG (Maguirre et al., 1991).

Tal y como se expusieron algunas de las limitantes en los datos, variables e indicadores, la georreferenciación de ellos, está influenciada por la cobertura, calidad y grado de actualización de las bases cartográficas existentes, tanto como por la coincidencia entre las unidades que colectan y almacenan datos (direcciones o unidades territoriales), y la estructura cartográfica disponible, o la compatibilidad de sus soportes. Por otra parte, aún con avances notables, ello continúa siendo una limitante en la ampliación del geoprocesamiento y en particular de los Sistemas de Información Geográfica en Salud.

La producción incansable de información de puntos, tornada rutina en el trabajo de vigilancia en salud y viabilizada por el uso de GPS y el georreferenciamiento de eventos de salud, puede aclarar los procesos ambientales y sociales involucrados en la producción de enfermedades, pero también convertirse en factor de complicación de los análisis necesarios, por el volumen, complejidad de los datos y la precisión de estos.

Al evaluar el escenario del geoprocesamiento en salud en varios países de nuestra región, Barcellos y Ramalho ( 2002), lo consideraron favorable teniendo en cuenta cuatro ejes de su desarrollo: La disponibilidad de bases de datos, los programas disponibles, el desarrollo tecnológico y la capacitación de personal. Transcurrida poco más de una década sin desconsiderar la validez de estas observaciones, las preocupaciones se trasladan hacia el propio proceso de georreferenciamiento de datos. En estudio a escala sub municipal en algunos territorios de Brasil, se constata que la calidad de los sistemas de información y de las bases cartográficas se incrementan, no obstante se aprecian dificultades que afectan la eficiencia y precisión de la localización de los eventos de salud y concluyen que la utilización más eficiente de las técnicas de geoprocesamiento depende de la disponibilidad y calidad de los datos georreferenciados.

Mucho más difícil de vencer, es el hecho de que el mapa representa "solo lo que se registra". Mientras entre territorios con escasos o sin, servicios de salud, se mantiene el llamado "silencio 
epidemiológico", en otros casos las tasas más elevadas de una determinada entidad en una unidad espacial, se obtiene donde la descentralización de determinados programas de control fue exitosa, o donde eventualmente se realizaron campañas de detección. Así los mapas pueden permitir identificar "causas epidemiológicas", ambientales, sociales, hereditarias y genéticas, y otros, o por el contrario las regularidades de la distribución hallada ser explicadas por "causas operacionales". Del otro lado de los sub registros, está la tarea de limpiar los sobre registros en las bases de datos disponibles.

Es indudable que la vigilancia en salud puede aumentar su sensibilidad y capacidad de detección y análisis de eventos de salud si logra incorporar el inmenso contenido de información de sistemas no formales, producidos al interior de las redes sociales virtuales (Boulos et al., 2011), sitios centinela (Flahault et al., 2006) y periódicos digitales en la web (Chunara et al., 2012). Estas informaciones son capaces de producir alertas tempranas para situaciones de riesgo, como los desastres naturales, al mismo tiempo en que constituyen herramientas para la movilización de la población y de entes gubernamentales.

En este sentido, la notificación de casos se convierte en una de las fuentes de información, que debe ser complementada y perfeccionada por asociación con otros sistemas de información como los registros de hospitalización, defunciones y datos de laboratorios clínicos (MerchanHamann e Laguardia, 2000).

Existe la posibilidad de representar en un mapa, eventos que ocurrieron a escasos minutos, en lo que se ha dado en llamar vigilancia en tiempo real. Proyección, coordenadas, técnica de representación, simbología, gama de colores, son obtenidos de forma automática. Se cuenta con proyecciones de forma automática, se calcula la escala de forma automática, se seleccionan las técnicas de representación de forma automática, los rangos de representación numéricos, gamas de colores, y el resto de los pasos clásicos cartográficos. Aunque se ha facilitado el manejo de datos espaciales, para que sean útiles, comunicativos e comprendidos, los mapas deben seguir os principios básicos y las normas de la Cartografía. Aunque se automatizan los análisis y que se creen nuevas herramientas, los SIG no dispensan el investigador, las teorías geográficas, ni el trabajo en el terreno. 
La búsqueda de uno o más mapas que sean representativos de un determinado problema de salud es un esfuerzo de profesionales de salud, cartografía, geografía, biología, entre otros, que pueden promover el intercambio de conceptos y técnicas de sus áreas de conocimiento.

\section{CONSIDERACIONES FINALES}

La Cartografía, Ciencia, Técnica y Arte, tiene cada vez más amplias opciones de contribuir a mejorar la salud de la población y al progreso de la equidad en salud. Se han abierto nuevas oportunidades para apoyar la identificación de determinantes de la salud, y la distribución óptima de servicios de atención. La manera de conducir estos análisis depende de la disponibilidad de datos y herramientas estadísticas pero, principalmente de la formulación de hipótesis y modelos que permitan rescatar la lógica de las relaciones entre los eventos de salud y las características del lugar, o de las relaciones entre la distribución de eventos de salud y la organización de los servicios de atención al evento dado.

Muchos de los que construyen y analizan mapas de salud en la actualidad, no hacen geografía de la salud o geografía médica, y pocas veces se menciona la cartografía, sustituida por el geoprocesamiento, la georeferenciación, o los abordajes espaciales en salud o en salud pública, o la cartografía es reducida a exponer sistemas geodésicos, de coordenadas o proyecciones.

Este hecho no puede valorase como negativo, y la dosis inicial de seducción por los mapas temáticos en salud, de la que emanaron múltiples documentos que ignoraban preceptos teóricos esenciales de la cartografía y de la teoría del problema que representa, se ha sustituido por sólidas contribuciones de diferentes profesionales que avanzan en la comprensión de la espacialidad de datos en salud, con técnicas avanzadas o sofisticadas, pero también con un actualizado conocimiento de la trama de determinantes que sustentan estas distribuciones (Iñiguez, 2007).

En un interesante artículo sobre los SIG y el análisis espacial, con el curioso título "No todos los mapas son iguales" Mika Rytkönen (2004) plantea que dependiendo de la cantidad y cualidad de los datos y de la metodología usada en el análisis un mapa puede ser "útil o engañoso". El 
peligro de las falsas pistas que un mapa de salud puede inducir tiene más de 20 años $^{6}$, y tal vez con cada vez más mapas ello se haya potenciado. Aunque este hecho pudiera aplicarse a la cartografía en general, en la de la salud y la Salud Pública tiene un significado esencial, porque su foco es la vida de las personas, y por tanto el curso de las sociedades. Los éxitos de su utilidad dependerán por tanto del rigor con el que se avance.

Sin dudas la cartografía en el campo de la salud y la Salud Pública progresa con pruebas de su contribución al conocimiento y la interpretación de eventos y procesos, de problemas y necesidades en salud. Queda ahora impulsar que los mapas que produce, sean soportes permanentes de acciones e intervenciones que minimicen los daños y respondan a las necesidades de salud de las poblaciones.

\section{REFERENCIAS}

Auchincloss AH, Gebreab SY, Mair C, Diez Roux AV. A review of spatial methods in epidemiology, 2000-2010. Annu Rev Public Health. 2013; 33: 107-122.

Barcellos C, Ramalho WM. Situação atual do geoprocessamento e da análise de dados espaciais em saúde no Brasil. Revista de Informática Pública 2002;4(2):221-230.

Barcellos C. Unidades y escalas en los análisis espaciales en salud. Revista Cubana de Salud Pública, 29(4): 307-313, 2003.

Barcellos, C. Detección de conglomerados espaciales de casos: La Geografía frente a una nube de puntos. In: Gustavo Buzai. (Org.). Métodos Cuantitativos en Geografía de la Salud. 1a ed. Buenos Aires- Lujan: Universidad Nacional de Lujan, 2007, v. 1, p. 19-25.

Boulos, MNK, Bernd Resch, DN Crowley, JG Breslin, G Sohn, R Burtner, WA Pike, E Jezierski, e KS Chuang. Crowdsourcing, Citizen Sensing and Sensor Web Technologies for Public and Environmental Health Surveillance and Crisis Management: Trends, OGC Standards and Application Examples. International Journal of Health Geographics 10, no. 1 (dezembro 21, 2011): 67.

\footnotetext{
${ }^{6}$ Cliff y Hagget 1992
} 
Briggs DJ, Elliott P (1995) The use of geographical information systems in studies on environment and health. World Health Stat Q 48: 85-94.

Buzai G D (2003) Mapas sociales urbanos. Lugar Editorial. Buenos Aires.

Cliff, A.D, Hagget, P (1992). Atlas of Disease Distributions. Cambridge, Blackwell.XiXii.

Curto de Casas, S. (2003) Mapas en Geografía Médica. Caderno Prudentino de Geografia. 5(117): 111-130.

Darchenkova. N. Fundamentos teóricos de la Geografía Médica. Conferencia dictada en abril de 1988 en el Instituto de Medicina Tropical Pedro Kourí. La Habana. Cuba (inédito). Cliff , A.D\& Hagget, P., (1992). Atlas of Disease Distributions. Cambridge, Blackwell. p Xi-Xii.

Dunn CE, Kingham SP, Rowlingson B, Bhopal RS, Cockings S, Foy CJ, Acquilla SD, Halpin J, Diggle P, Walker D (2001) Analysing spatially referenced public health data: a comparison of three methodological approaches. Health and Place 7: 1-12.

Fisher, Rohan P, e BA Myers. Free and simple GIS as appropriate for health mapping in a low resource setting: a case study in eastern Indonesia. International Journal of Health Geographics 10 (2011): 15.

Gatrell AC, Bailey TC, Diggle PJ, Rowlingson BS (1996) Spatial point pattern analysis and its application in geographical epidemiology. Trans Inst Br Geogr 21: 256-274.

Goldstein, R.A; Christovam Barcellos; Monica de Avelar Figueiredo Mafra Magalhães; Renata Gracie; Francisco Viacava.(2013) A experiência de mapeamento participativo para a construção de uma alternativa cartográfica para a ESF. Ciênc. Saúde Coletiva. 18(1).

Iñiguez R.L. (2007) Seducción de los mapas temáticos en salud y las limitantes de la dimensión cuantitativa. En: Buzai,G (org) Métodos Cuantitativos en Geografía de la Salud. Luján, Argentina. Universidad de Luján. 
Iñiguez, R.L, Barcellos, C \& Peiter, P. (1999) Utilização dos mapas no campo da Epidemiologia no Brasil: reflexões sobre trabalhos apresentados no IV Congresso Brasileriro de Epidemiología. Informe Epidemiológico do SUS. Vol 8-N 2. Abri/Jun 1999.

King PE (1979) Problems of spatial analysis in geographical epidemiology. Social Science \& Medicine. 13: 249-252.

Kulldorff M, Nagarwalla N (1995) Spatial disease clusters: detection and inference. Stat Med 14: 799-810.

Levine N (2002) CrimeStat: A spatial statistics program for the analysis of crime incident locations. Ned Levine \& Associates, Houston, TX, and the National Institute of Justice. Washington, DC.

Michelozzi P, Capon A, Kirchmayer U, Forastiere F, Biggeri A, Barca A, Perucci CA. (2002) Adult and childhood leukemia near a high-power radio station in Rome, Italy. American Journal of Epidemiology. 155(12):1096-103.

Mika JP Rytkönen (2004) Not all maps are equal: GIS and spatial analysis in epidemiology. International Journal of Circumpolar Health 63:1

Openshaw S (1984) Ecological fallacies and the analysis of areal census-data. Environment \& Planning A. 16(1): 17-31.

Openshaw S (1994) Two exploratory space-time attribute pattern analyzers relevant to GIS. In S. Fotheringham and P. Rogerson, editors, Spatial Analysis and GIS. London: Taylor and Francis.

Rushton G (2003) Public health, GIS, and spatial analytic tools. Annu Rev Public Health 24: 43-56.

Sáenz de la Calzada, C., 1956. Los fundamentos de la Geografía Médica. Boletín de la Sociedad Mexicana de Geografía y Estadística. Tomo LXXXI :I

Santos M (1997) A natureza do espaço: técnica e tempo, razão e emoção. 2. Ed. São Paulo: Hucitec, $273 p$. 
Santos SM, Barcellos C, Carvalho MS, Flores R (2001) Detecção de aglomerados espaciais de óbitos por causas violentas em Porto Alegre, Rio Grande do Sul, Brasil. Cad Saúde Pública 17: 1141-1151.

Sorre, M (1978) Principes de Cartographie appliqué a l'ecologie humaine. Soc.Sxi\& Med, Vol.12D 238-250, Pergamon Press.

Snow J (1989) Sobre a maneria de transmissão do cólera. HUCITE-ABRASCO São Pãulo-Rio de Janeiro. 1990. 249:134-142

Stampach, R; Kubicek, P; \& Geryk 2010 Cartographic aspects of health databases visualization in global context. 3rd ISDE DIGITAL EARTH SUMMIT 12-14 June, Nessebar, Bulgaria.

Susser M, Susser E (1996) Choosing a future for epidemiology: I. Eras and paradigms. American Journal of Public Health. 86(5): 668-673.

Waller LA (2000) A civil action and statistical assessments of the spatial pattern of disease: do we have a cluster? Regul Toxicol Pharmacol 32: 174-183. 